\title{
DETERMINAÇÃO DAS ISOTERMAS DE ADSORÇÃO E DO CALOR DE SORÇÃO DA FARINHA DE BABAÇU COMERCIAL
}

\author{
R. M. SAMPAIO ${ }^{1}$, E. N. V. MATOS ${ }^{2}$ e M. L. de PAULA ${ }^{3}$ \\ ${ }^{1}$ Universidade Federal do Maranhão, Departamento de Tecnologia Química \\ ${ }^{2}$ Universidade Federal do Maranhão, Aluna do Curso de Engenharia Química \\ ${ }^{3}$ Universidade Federal do Maranhão, Departamento de Farmácia \\ E-mail para contato: romildosampaio@uol.com.br
}

\begin{abstract}
RESUMO - O babaçu (Orbignya martiana), palmeira abundante no Nordeste brasileiro, apresenta grande potencial econômico nas regiões em que é produzida. Seu fruto é dividido em quatro partes: epicarpo, mesocarpo, endocarpo e amêndoa, todos com aplicações industriais e comerciais. Sua farinha, obtida a partir do mesocarpo, tem tido crescente aceitação no setor alimentício, sem, no entanto, apresentar na literatura muitos estudos a respeito de suas isotermas de sorção, importantes para predizer a estabilidade química e física em função do conteúdo de água e o teor de umidade que impede o crescimento dos microrganismos. Assim, os objetivos deste trabalho foram estudar o comportamento das isotermas de adsorção da farinha de babaçu comercial às temperaturas de 25 e $40^{\circ} \mathrm{C}$, selecionar o modelo que melhor se ajusta aos dados experimentais e estimar o calor isostérico de sorção. As isotermas foram construídas pelo método gravimétrico. Para os ensaios de adsorção, foram empregadas amostras de farinhas de babaçu previamente secas. A seleção do melhor modelo foi feita com base no coeficiente de determinação e no qui-quadrado. À exceção de BET, todos os demais modelos testados permitiram ajustes muito bons nas temperaturas estudadas, sendo que a $25{ }^{\circ} \mathrm{C}$ os modelos de Henderson e Peleg apresentaram os melhores ajustes, enquanto que a $40^{\circ} \mathrm{C}$, o de GAB foi o mais adequado. Os calores isostéricos de adsorção apresentaram comportamento inversamente proporcional à umidade de equilíbrio.
\end{abstract}

\section{INTRODUÇÃO}

O babaçu (Orbignya martiana) é uma palmeira abundante no nordeste brasileiro. A maior concentração dessas palmeiras encontra-se no Maranhão, representando cerca de $80 \%$ da produção nacional de babaçu. O fruto desta palmeira é totalmente aproveitável, constituindo assim um produto de grande potencial econômico, social e cultural. Dentre os diversos produtos do babaçu encontra-se a farinha obtida do mesocarpo, sendo este caracterizado por seu elevado teor de amido (50\% p) e fibras (10\% p) (Silva, 2011).

A farinha é obtida a partir da secagem e trituração do mesocarpo. O mesocarpo transformado em pó é peneirado, umedecido e finalmente torrado em fogo alto. $\mathrm{O}$ interesse na farinha de mesocarpo de babaçu está pautado nas suas propriedades medicinais, tais como anti-inflamatória alterações nas 


\section{9 a 22 de outubro de 2014 \\ Florianópolis/SC}

funções gastrointestinais, redução nos níveis de colesterol, glicemia e insulina pós-prandial (Baldez et al., 2006). Contudo, mesmo apresentando excelentes propriedades há uma deficiência de estudos que tratem desse subproduto do babaçu.

A água presente nos alimentos é o principal meio para o crescimento microbiano; além disso, funciona como solvente nas transformações químicas e bioquímicas. $\mathrm{O}$ termo atividade de água $\left(\mathrm{A}_{\mathrm{w}}\right)$ sugere um indicativo da quantidade de moléculas livres ou ativas de água disponíveis para ocasionar deterioração das propriedades dos alimentos (Labuza, 1997).

O controle da umidade relativa do ar é fundamental na garantia da estabilidade do produto, pois quando a pressão de vapor da água na superfície do material não é igual a do ar que a envolve ocorre uma transferência de massa a fim de alcançar o equilíbrio. $\mathrm{O}$ equilíbrio pode ser alcançado no ganho ou na perda de umidade. $\mathrm{O}$ primeiro caso refere-se à adsorção e o segundo à dessorção (Resende et al., 2006).

As isotermas de sorção são ferramentas úteis para prever a estabilidade de alimentos em diversas condições atmosféricas. Elas fornecem uma relação do conteúdo de umidade com a umidade relativa ou atividade de água numa temperatura específica. A informação obtida com as isotermas é fundamental na formulação de misturas de alimentos evitando a migração de água entre os diversos ingredientes, na determinação da permeabilidade requerida no material embalado, na determinação do teor de umidade que impede o crescimento dos microrganismos de interesse e na predição da estabilidade química e física dos alimentos em função do conteúdo de água (Oliveira, 2008). O comportamento das isotermas de sorção pode ser previsto a partir do ajuste dos dados experimentais por modelos propostos por diversos autores, que são úteis no conhecimento das características dos produtos (Silva, 2007).

O calor isostérico de sorção é a energia necessária para romper as forças de ligação entre a água e a superfície da substância adsorvente (Resende et al., 2006).

O pouco estudo sobre as isotermas de sorção da farinha de babaçu foram a motivação maior deste trabalho, cujos objetivos principais foram estudar o comportamento e modelar as isotermas de adsorção da farinha de babaçu comercial às temperaturas de 25 e $40^{\circ} \mathrm{C}$ e calcular o seu calor isostérico de adsorção.

\section{MATERIAL E MÉTODOS}

Os experimentos foram conduzidos no Laboratório de Petróleo e Sistemas Complexos da Universidade Federal do Maranhão. A farinha de mesocarpo de babaçu foi adquirida em um único lote da mesma marca no comércio local de São Luís. Inicialmente, a farinha foi levada à estufa em uma temperatura de $60^{\circ} \mathrm{C}$ por 8 horas, para eliminação de toda água presente.

As isotermas de adsorção foram obtidas pelo método gravimétrico estático. Para determinação da atividade de água, foram empregadas soluções salinas saturadas de nove tipos de sais, conforme as equações apresentadas no trabalho de Raji e Ojediram (2011). A Tabela 1 apresenta os sais 
empregados e suas respectivas atividades de água $\left(\mathrm{A}_{\mathrm{w}}\right)$ nas temperaturas de 25 e $40^{\circ} \mathrm{C}$.

As soluções salinas saturadas, após preparadas foram acondicionadas em potes hermeticamente fechados, de maneira que formassem uma camada de sal e uma fina película de água acima desta. Amostras contendo aproximadamente $0,5 \mathrm{~g}$ de farinha isenta de umidade foram colocadas, inicialmente, em forminhas de papel laminado e depois no interior do pote sobre um suporte, que as manteve acima da solução.

Para determinação da atividade de água, as amostras foram pesadas diariamente em balança analitica até que a massa tivesse variação máxima de $\pm 0,001 \mathrm{~g}$. Após terem suas umidades determinadas e relacionadas com as atividades de água correspondentes, as isotermas de adsorção foram ajustadas matematicamente, empregando análise de regressão não-linear, com o auxílio do Origin 8.0. De acordo com a Tabela 2, foram testados cinco modelos largamente empregados na literatura para determinação de isotermas de alimentos: Brunauer, Emmett e Teller (BET), Gugghenheim, Anderson e de Boer (GAB), Oswin, Henderson e Peleg (Sampaio et al., 2009).

Os calores isostéricos de adsorção $\left(Q_{s t}\right)$ foram determinados usando a equação de ClasiusClapeyron (Equação 1).

Tabela 1 - Sais empregados e suas respectivas atividades de água às temperaturas de 25 e $40^{\circ} \mathrm{C}$

\begin{tabular}{cccccc}
\hline $\mathrm{Sal}$ & $\mathrm{A}_{\mathrm{w}}\left(25^{\circ} \mathrm{C}\right)$ & $\mathrm{A}_{\mathrm{w}}\left(40^{\circ} \mathrm{C}\right)$ & $\mathrm{Sal}$ & $\mathrm{A}_{\mathrm{w}}\left(25^{\circ} \mathrm{C}\right)$ & $\mathrm{A}_{\mathrm{w}}\left(40^{\circ} \mathrm{C}\right)$ \\
$\mathrm{MgCl}_{2}$ & 0,328885 & 0,313226 & $\mathrm{NaCl}$ & 0,761997 & 0,734455 \\
$\left.\mathrm{Mg} \mathrm{NO}_{3}\right)_{2}$ & 0,536142 & 0,506261 & $\mathrm{NaBr}$ & 0,574593 & 0,535099 \\
$\mathrm{~K}_{2} \mathrm{CO}_{3}$ & 0,443338 & 0,433120 & $\mathrm{CH}_{3} \mathrm{COOK}$ & 0,237063 & 0,206397 \\
$\mathrm{LiCl}$ & 0,114296 & 0,105450 & $\mathrm{KI}$ & 0,689900 & 0,662000 \\
$\mathrm{KCl}$ & 0,855123 & 0,806039 & & & \\
\hline
\end{tabular}

Tabela 2 - Modelos usados para ajuste de isotermas de sorção de alimentos.

\begin{tabular}{cc}
\hline Nome do modelo & Modelo \\
Gugghenheim, Anderson e de Boer (GAB) & $X=\frac{X a_{o} C K a_{w}}{\left(1-K a_{w}\right)\left(1-K a_{w}+C K a_{w}\right)}$ \\
Brunauer, Emmett, Teller (BET) & $\frac{a_{w}}{\left(1-a_{w}\right) \cdot X}=\frac{1}{X X_{o} C}+\frac{(C-1)}{X{ }_{o} C} a_{w}$ \\
Oswin & $\mathrm{X}=\mathrm{B}_{2}\left[a_{\mathrm{w}} /\left(1-a_{\mathrm{w}}\right)\right]^{\mathrm{B} 1}$ \\
Henderson & $\left(1-a_{\mathrm{w}}\right)=\exp \left[-\mathrm{B}_{2}(\mathrm{X})^{\mathrm{B} 1}\right]$ \\
Peleg & $X=a a_{w}{ }^{b}+c a_{w}{ }^{d}$ \\
\hline
\end{tabular}


onde: $\mathrm{X}$ é o conteúdo de umidade $(\mathrm{kg} / \mathrm{kg}) ; \mathrm{X}_{\mathrm{o}}$ é conteúdo da camada monomolecular $(\mathrm{kg} / \mathrm{kg}) ; \mathrm{aw}$ é a atividade de água e; $\mathrm{B}_{1}, \mathrm{~B}_{2}, \mathrm{C}, \mathrm{K}, a, b, c$ e $d$ são constantes das equações

$$
\ln \left(\frac{a w_{1}}{a w_{2}}\right)=\frac{q_{s t}}{R}\left(\frac{1}{T_{1}}-\frac{1}{T_{2}}\right)
$$

\section{RESULTADOS E DISCUSSÃO}

As isotermas de sorção foram construídas a partir dos valores de atividade de água de cada sal utilizado e dos seus respectivos dados de teor de umidade adsorvido. Foram descartadas todas as amostras que apresentaram desenvolvimento de fungos, especialmente a altos valores de atividade de água. A importância dessas curvas está relacionada com a possibilidade de avaliar o comportamento da farinha de babaçu em diferentes ambientes que incluem mudança de umidade relativa e temperatura.

A Figura 1-a apresenta a isoterma de adsorção da farinha de babaçu a $25^{\circ} \mathrm{C}$ e a Figura 1-b a 40 ${ }^{\circ} \mathrm{C}$. Como esperado, as amostras que mais absorveram umidade foram aquelas postas em contato com as soluções salinas com maiores atividades de água $\left(\mathrm{A}_{\mathrm{w}}\right)$. Para os ensaios de adsorção a $40^{\circ} \mathrm{C}$ observou-se um aumento na atividade de água para um mesmo teor de umidade, para a maioria dos sais, quando comparado aos dados obtidos a $25^{\circ} \mathrm{C}$, mostrando a esperada dependência da umidade de equilíbrio em relação à temperatura.

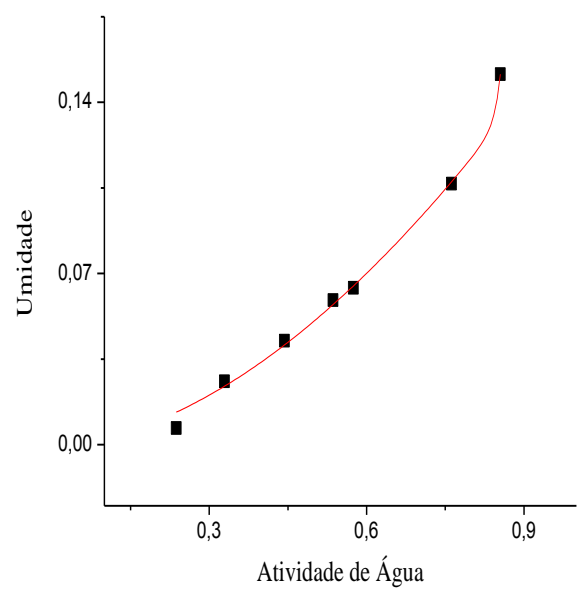

(a)

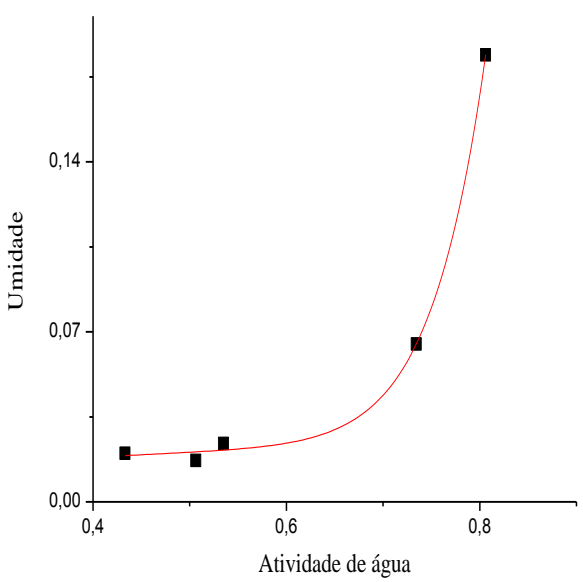

(b)

Figura 1 - Isoterma de adsorção nas temperaturas de $25^{\circ} \mathrm{C}$ (a) e $40^{\circ} \mathrm{C}$ (b) 
Os modelos testados apresentaram bons ajustes para a adsorção da farinha de babaçu nas duas temperaturas estudadas. $\mathrm{O}$ bom ajuste obtido com o modelo de GAB confirma descrições da literatura que afirmam que o mesmo ajusta bem a grande maioria das isotermas de alimentos. $\mathrm{O}$ modelo de BET, que segundo Kurozawa et al. (2005) não é aconselhável em valores de $\mathrm{A}_{\mathrm{w}}$ superiores a 0,45, apresentou os menores valores para o coeficiente de determinação, especialmente para a temperatura de $40{ }^{\circ} \mathrm{C}$. As duas isotermas apresentaram comportamento que se assemelham àqueles previstos para uma curva de adsorção do tipo S, segundo descrição de Giles (Al-Munhtaseb et al., 2004). Este comportamento é mais associado a adsorção de alimentos dada as características que os mesmos possuem de absorver grandes concentrações de água com baixa concentração de soluto.

As Tabelas 3 e 4 apresentam os valores dos parâmetros e os coeficientes estatísticos da adsorção a $25{ }^{\circ} \mathrm{C}$ e $40{ }^{\circ} \mathrm{C}$, respectivamente. Os modelos de Henderson e de Peleg proporcionaram o melhor ajuste para a adsorção a $25^{\circ} \mathrm{C}$, enquanto que à temperatura de $40{ }^{\circ} \mathrm{C}, \mathrm{GAB}$ foi o melhor modelo. De acordo com o ajuste obtido para o modelo de GAB, o conteúdo de umidade da camada monomolecular $\left(\mathrm{X}_{0}\right)$ foi de $0,0582 \mathrm{~kg}$ água $/ \mathrm{kg}$ material seco para o experimento de adsorção a $25^{\circ} \mathrm{C}$ e $0,00845 \mathrm{~kg}$ água $/ \mathrm{kg}$ material seco para a adsorção a $40^{\circ} \mathrm{C}$. Em produtos alimentícios esse valor é um indicativo da estabilidade do alimento frente à maioria das transformações físicas e químicas indesejáveis.

Os resultados do coeficiente de determinação e do teste qui-quadrado indicam que, com exceção de BET, todos os demais modelos testados apresentaram bons ajustes para a adsorção da farinha de babaçu a $25^{\circ} \mathrm{C}$ e a $40^{\circ} \mathrm{C}$. A curva do calor isostérico de adsorção, apresentada na Figura 3, apresenta um decréscimo na energia liberada no processo adsortivo quando a umidade de equilíbrio aumenta. Os valores do calor isostérico de adsorção (Qst) obtidos para a farinha de babaçu situaram-se entre 179,7 e $1063,2 \mathrm{~kJ} / \mathrm{kg}$.

Tabela 3 - Parâmetros de ajuste da isoterma de adsorção da farinha de babaçu comercial para os diferentes modelos testados à temperatura de $25^{\circ} \mathrm{C}$

\begin{tabular}{|c|c|c|c|c|c|c|}
\hline \multirow{3}{*}{$\begin{array}{l}\text { Modelo } \\
\text { GAB }\end{array}$} & \multicolumn{3}{|c|}{ Parâmetros dos Modelos } & & \multicolumn{2}{|c|}{ Parâmetros Estatísticos } \\
\hline & & & & & $\mathrm{R}^{2}$ & $x^{2}$ \\
\hline & $\begin{array}{c}\mathrm{X}_{0} \\
0,0582\end{array}$ & $\begin{array}{c}\mathrm{C}_{\mathrm{GAB}} \\
1,4245\end{array}$ & $\begin{array}{c}\mathrm{K}_{\mathrm{GAB}} \\
0,82051\end{array}$ & & 0,9904 & 0,0000224 \\
\hline BET & $\begin{array}{c}\mathrm{X}_{0} \\
0.0235\end{array}$ & $\begin{array}{l}\mathrm{C}_{\mathrm{BET}} \\
-100\end{array}$ & & & 0,9261 & 0,0001724 \\
\hline Oswin & B1 & B2 & & & 0,9809 & 0,0000445 \\
\hline Henderson & $\begin{array}{c}0,6496 \\
\mathrm{~B} 1^{\mathrm{H}} \\
0,91961\end{array}$ & $\begin{array}{c}0,0486 \\
\mathrm{~B} 2^{\mathrm{H}} \\
11,025\end{array}$ & & & 0,9919 & 0,0000188 \\
\hline Peleg & $\begin{array}{c}\mathrm{a} \\
3434,6043\end{array}$ & $\begin{array}{c}\mathrm{b} \\
77,24581\end{array}$ & $\begin{array}{c}\mathrm{c} \\
0,17498 \\
\end{array}$ & $\begin{array}{c}\mathrm{d} \\
1,79079\end{array}$ & 0,9927 & 0,0000178 \\
\hline
\end{tabular}


Tabela 4 - Parâmetros de ajuste da isoterma de adsorção da farinha de babaçu comercial para os diferentes modelos testados à temperatura de $40^{\circ} \mathrm{C}$

\begin{tabular}{|c|c|c|c|c|c|c|}
\hline \multirow[t]{2}{*}{ Modelo } & \multicolumn{3}{|c|}{ Parâmetros dos Modelos } & & \multicolumn{2}{|c|}{ Parâmetros Estatísticos } \\
\hline & & & & & $\mathrm{R}^{2}$ & $\mathrm{x}^{2}$ \\
\hline \multirow[t]{2}{*}{ GAB } & $\mathrm{X}_{0}$ & $\mathrm{C}_{\mathrm{GAB}}$ & $\mathrm{K}_{\mathrm{GAB}}$ & & 0,9984 & 0,0000083 \\
\hline & 0,0084 & 1010 & 1,1837 & & & \\
\hline \multirow[t]{2}{*}{ BET } & $\mathrm{X}_{0}$ & $\mathrm{C}_{\mathrm{BET}}$ & & & 0,8442 & 0,0000785 \\
\hline & 0,3424 & 0,0500 & & & & \\
\hline \multirow[t]{2}{*}{ Oswin } & B1 & B2 & & & 0,9626 & 0,000188 \\
\hline & 2,0505 & 0,0097 & & & & \\
\hline \multirow[t]{2}{*}{ Henderson } & $\mathrm{B} 1^{\mathrm{H}}$ & $\mathrm{B} 2^{\mathrm{H}}$ & & & 0,9459 & 0,000272 \\
\hline & 0,2535 & 2,5336 & & & & \\
\hline \multirow[t]{2}{*}{ Peleg } & $\mathrm{a}$ & $\mathrm{b}$ & $\mathrm{c}$ & d & 0,9958 & 0,0000209 \\
\hline & 0,0277 & 0,4490 & 3,6906 & 14,5871 & & \\
\hline
\end{tabular}

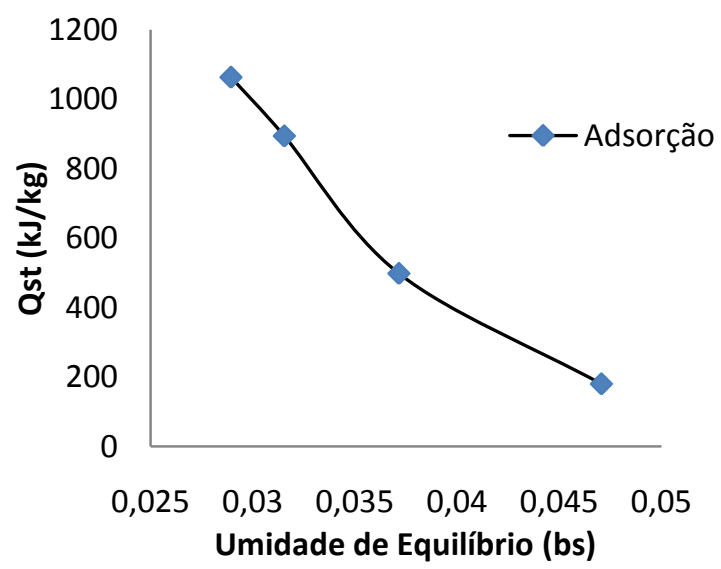

Figura 3 - Calor isostérico de adsorção em função da umidade de equilíbrio da farinha de babaçu.

\section{CONCLUSÕES}

Os resultados obtidos indicaram que foi possível obter ajustes muito bons dos dados experimentais do processo de adsorção da farinha de babaçu comercial às temperaturas de $25 \mathrm{e}$ $40^{\circ} \mathrm{C}$ pelos modelos de GAB, Henderson, Oswin e Peleg.

A diferença entre os valores do conteúdo de umidade da camada monomolecular $\left(\mathrm{X}_{0}\right)$, obtidos pelos modelos de GAB e de BET, devem-se principalmente a limitação que o modelo de BET possui para ajustar dados com altos teores de atividade de água, o que gerou os piores ajustes entre todos os modelos testados nas duas temperaturas estudadas.

Os calores isostéricos de sorção apresentaram grande variação nos valores obtidos, sendo estes 
maiores quanto menores foram as umidades de equilíbrio.

\section{REFERÊNCIAS}

AL-MUNHTASEB, A. H.; MCMINN, W. A. M.; MAGEE, T. R. A. Water sorption isotherms of starch powders. Part 1: mathematical description of experimental. J. Food Eng., v. 61, n.1, p. 297-307, 2004.

BALDEZ, R. N.; MALAFAIA, O.; CZECZKO, N. G. Análise da cicatrização do cólon com uso do extrato aquoso da Orbignya phalerata (Babaçu) em ratos. Acta Cirúrgica Brasileira, v.21, 2006.

KUROZAWA, L.E.; EL-AOUAR, A. A.; MURR, F. E. X. Obtenção de Isotermas de Dessorção de Cogumelo In Natura e Desidratado Osmoticamente. Ciênc. Tecnol. Aliment., vol. 25, n. 4, 2005.

LABUZA, T. P. The properties of water in relationship to water binging in food: a review. $J$. Food Proces. Preserv. v. 1, n. 2, p. 167- 190, 1997.

OLIVEIRA, F.C. Estudos tecnológicos e de engenharia para o armazenamento e processamento do pinhão. Porto Alegre: UFRGS, 2008. Tese (Doutorado em Engenharia).

RAJI, A. O.; OJEDIRAN, J. O. Mositure sorption isotherms of two varieties of Miller. Food Bioprod. Process., v. 89, n. 3, p. 178-184, 2011.

RESENDE, O.; CORRÊA, P.C.; GONELI, A.L.; RIBEIRO, D. M. Isotermas e calor isostérico de sorção do feijão. Ciênc. Tecnol. Aliment., Campinas, v. 26, n. 3, p. 626-631, 2006.

SAMPAIO, R. M.; MARCOS, S. K.; MOARES, I. C. F.; PEREZ, V. H. Moisture adsorption behavior of biscuits formulated using wheat, oatmeal and passion fruit flour. J. Food Proces. Preserv., v. 33, p. 105-113, 2009.

SILVA, A. P. S. Caracterização físico-química e toxicológica do pó de mesocarpo do babaçu (Orbignyaphalerata Mart): subsídio para o desenvolvimento de produtos. Teresina: UFPI, 2011. Dissertação (Mestrado em Ciências Farmacêuticas).

SILVA, F.; PARK, K. J.; MAGALHÃES, P.M. Isotermas de dessorção de Calendula officinalis L.: determinação experimental e modelagem matemática. Rev. Bras. Pl. Med., Botucatu, v.9, n.1, p.21-28, 2007. 